\title{
Identification of the Lumbar Interspinous Spaces by Palpation and Verified by X-rays
}

\author{
Kei Tanaka* ${ }^{1}$, Shingo Irikoma ${ }^{1}$, Sotaro Kokubo ${ }^{1}$
}

1. Department of Anesthesiology, Seirei Hamamatsu General Hospital, Hamamatsu, Japan. Received from the Department of Anesthesiology, Seirei Hamamatsu General Hospital, Hamamatsu, Japan.

Submitted on March 22, 2012. Approved on April 23, 2012.

\author{
Keywords: \\ Anesthesia, Obstetrical; \\ Cesarean section; \\ Analgesia, Epidural; \\ Palpation; \\ Radiography.
}

\begin{abstract}
Background and objectives: Palpation has been shown to be rather inaccurate at identifying lumbar interspinous spaces in neuraxial anesthesia. The aim of this study is to assess the accuracy of the determination of the lumbar interspinous spaces by anesthesiologist's palpation using postoperative X-rays in obstetric patients.

Methods: We reviewed the anesthetic record and the post-operative abdominal X-rays of the cesarean sections. We indwelled the epidural catheter for post-operative one-shot analgesia. We included combined spinal and epidural anesthesia cases and compared the interspinous level which the anesthesiologist recorded and the epidural catheter insertion level confirmed by abdominal X-ray for each case. We also evaluated the factors (age, body weight, height, Body Mass Index, gestational age, and the type of surgery [planned / emergency]) leading to misidentification of interspinous level.

Results: Nine hundred and sixty seven cesarean sections were performed and a total of 835 cases were evaluated. The levels of the puncture documented by the anesthesiologists were in agreement with the actual catheter insertion levels in $563(67 \%)$ cases. When the anesthesiologists aimed at L2-3 level, we found the catheter insertion at L1-2 in 5 cases (4.9\%), none of which had any post-operative neurological deficits. No variables evaluated were significantly associated with misidentification of interspinous level by the anesthesiologists.

Conclusions: There was a discrepancy between the anesthesiologists' estimation by palpation and the actual catheter insertion level shown in X-rays. It seems to be safer to choose the interspinous level L3-4 or lower in spinal anesthesia.

○ 2013 Sociedade Brasileira de Anestesiologia. Published by Elsevier Editora Ltda. All rights reserved.
\end{abstract}

\section{Introduction}

Palpation has been shown to be rather inaccurate at identifying lumbar interspinous spaces in neuraxial anesthesia. A needle placement at a high interspinous level in spinal anesthesia or combined spinal-epidural anesthesia (CSEA) can lead to iatrogenic neurologic deficits. There are some reports of neurological injury when the vertebral level is misidentified during the spinal block ${ }^{1-3}$.

*Corresponding author: 430-8558 2-12-12 Sumiyoshi, Hamamatsu, Shizuoka, Japan.

E-mail: Kei49tanaka@gmail.com

ISSN/\$ - see front metter c 2013 Sociedade Brasileira de Anestesiologia. Published by Elsevier Editora Ltda. All rights reserved. 
Many studies have tried to confirm the accuracy of lumbar interspinous space levels identification by anesthesiologist's palpation ${ }^{4-10}$, but were limited by a small number of subjects. Furthermore, few studies used radiography as the imaging technique to verify the estimation level.

This study was designed to examine the accuracy of the anesthesiologist's palpation for identifying the lumbar interspinous level in obstetric patients, using post-operative X-rays.

\section{Materials and Methods}

We retrospectively reviewed the anesthetic records and the post-operative abdominal X-rays of the cesarean sections performed at Seirei Hamamatsu General Hospital between January 2007 and December 2008. Our institutional review board approved this study (Seirei Hamamatsu General Hospital).

Patients were placed in the right lateral decubitus position with flexed knees and hips. We used Portex CSEA needle (PX100, Smiths Medical ${ }^{\circledR}$, USA) for single space segment technique. We have indwelt epidural catheter (EC-5500, Arrow ${ }^{\circledR}$, USA) which is visible on X-ray for post-operative one-shot analgesia. Anesthesiologists usually used Jacoby's line (Tuffier's line) as landmark crossing the L4 spinous process. In our institution, an abdominal X-ray is taken routinely before every patient is discharged from the operating room for post-operative check-up. We included CSEA cases and compared the interspinous level that the anesthesiologist recorded and the actual epidural catheter insertion level confirmed by abdominal X-ray for each case (Figure 1).

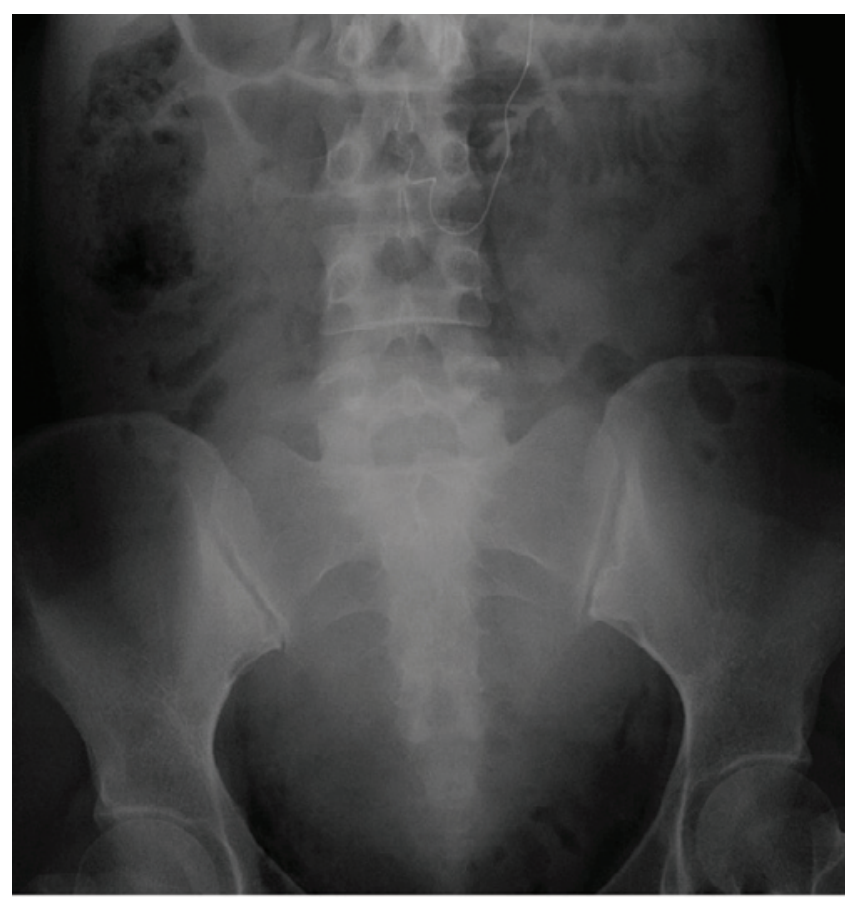

Figure 1 - The post-operative abdominal X-ray.

The epidural catheter appears inserted at the L2-3 interspace.
The primary outcome was the level of agreement between the lumbar interspinous level identified by palpation and the epidural catheter insertion level. The strength of the agreement between palpation and catheter level was assessed by unweighted Kappa statistic. The agreement is considered almost perfect for Kappa value $>0.81$, substantial for Kappa value between 0.61 and 0.80, moderate for Kappa value between 0.41 and 0.60 , fair for Kappa value between 0.21 and 0.41 , and poor for Kappa value $<0.20$.

The secondary outcome was to evaluate the factors leading to misidentification of interspinous level. The factors included the subject's demographics (age, body weight, height, Body Mass Index, and gestational age at surgery) and the type of surgery (planned or emergency). Continuous variables were analyzed using t-test or Mann-Whitney-U test and categorical variables were analyzed using Chi-square test. Multiple logistic regression was used for multivariate analysis. Data were examined using a statistics program (SPSS Statistics ver19, IBM®, JAPAN) with $p$ value $<0.05$ regarded as statistically significant.

\section{Results}

Nine hundred and sixty seven cesarean sections were performed during the study period. One hundred and thirty two cases were excluded because the catheter was not used (general anesthesia or single-shot spinal anesthesia) or was difficult to identify in the abdominal X-ray. A total of 835 cases were evaluated. Table 1 describes the demographic details of the study subjects.

The levels of the puncture documented by the anesthesiologists were in agreement with the actual catheter insertion levels in $563(67 \%)$ cases (Figure 2). The strength of this agreement (measured by unweighted Kappa) was 0.275, which represents 'fair' agreement $(p<0.01)$.

In 238 cases (29\%), the catheter insertion level seen in the X-ray was one interspace higher than the level documented in the anesthetic record; the catheter insertion level was one interspace lower in 34 cases $(4 \%)$.

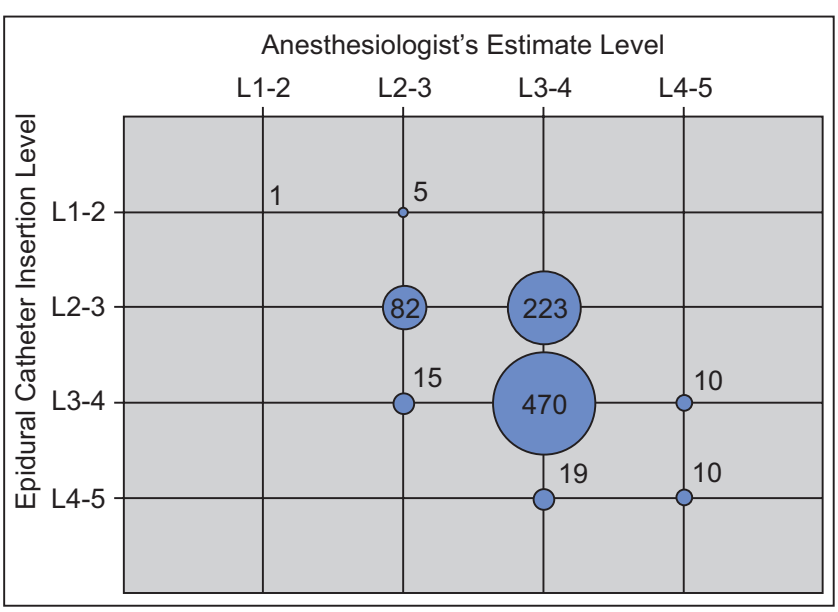

Figure 2 Comparison between interspinous levels estimated by palpation and actual catheter insertion levels in X-rays. The numbers in the circles describe the number of cases. 
Table 1 Demographic Data.

\begin{tabular}{ll}
\hline Age & $32(19-48)$ \\
Body Weight $(\mathrm{kg})$ & $60.9(40.0-107.2)$ \\
Height $(\mathrm{cm})$ & $157(138-174)$ \\
Body Mass Index $\left(\mathrm{kg} \cdot \mathrm{m}^{-2}\right)$ & $24.7(15.6-44.9)$ \\
Gestational Age at delivery (week) & $38(23-42)$ \\
Type of surgery & \\
\multicolumn{1}{l}{ Planned } & $650(78)$ \\
$\quad$ Emergency & $185(22)$ \\
\hline
\end{tabular}

Values are Median (range) or Number (\%).

When anesthesiologists aimed at L2-3 interspinous level (105 cases), we found the catheter insertion at L1-2 in 5 cases $(4.9 \%)$, none of which had any post-operative neurological deficits.

No factors evaluated by univariate and multivariate logistic regression were statistically associated with misidentification of interspinous level (Table 2).

\section{Discussion}

In this study, we confirmed a discrepancy between the lumbar interspinous level identified by palpation and the actual epidural catheter insertion level.

Previous studies demonstrated accuracy in 29-59\% of subjects when attempting to identify lumbar interspaces by palpation ${ }^{3-9}$. Our success rate $(67 \%)$ was higher than those smaller size studies. The reason may be that our study population - Japanese pregnant women - showed lower median Body Mass Index than other studied populations.

Several imaging techniques such as ultrasound and MRI have been used to verify the interspinous level. Broadbent et al. ${ }^{6}$ assessed the accuracy of palpation using MRI in a study of 100 patients. Many studies have compared palpation to ultrasound imaging ${ }^{7-10}$. With lumbar radiograph as the standard, Furness et al. ${ }^{7}$ showed that the correct interspace was identified in $71 \%$ of 50 cases using ultrasound, compared with a $30 \%$ success rate with palpation. Schlotterbeck et al. ${ }^{9}$ examined the needle scar position by ultrasound and compared with the anesthetic record. Van Gessel et al. ${ }^{5}$ used a spinal catheter and radiography to evaluate the accuracy of palpation in a small study of 29 patients, reporting the success rate of $41 \%$. The subjects of their study were all elderly patients with a mean age of 85 years old. To our knowledge, our study, involving 835 cases, is the largest clinical study using radiographic imaging to confirm the actual interspinous level identified by palpation in obstetric anesthesia.

Our study also confirmed that actual punctured level was often more cephalad than the palpation estimate when there was disagreement, which is consistent with previous studies ${ }^{6,8-10}$. This tendency was marked when the anesthesiologists aimed at the L3-4 or L4-5 interspinous levels.

Although the anesthesiologists showed the highest accuracy of $80.4 \%$ when aiming at L2-3, we found about $5 \%$ of error of needle placement at L1-2 interspinous level. A series of anatomical dissection studies demonstrated that 28-58\% of adult cords end below the body of L1 ${ }^{11-13}$. In order to prevent spinal cord trauma, it is not recommended to attempt insertion above L3-4 interspace routinely. It seems to be safer to choose the interspinous level L3-4 or lower if a choice of suitable interspaces exits.

No risk factors were derived as predictive of misidentification of interspinous level in this study, although several factors such as Body Mass Index, spinal abnormality, and anatomical landmark quality have been shown to be associated with difficult neuraxial block ${ }^{14}$.

Our study verified the accuracy of identification of the lumbar interspinous level by palpation and did not search for an alternative technique to improve the accuracy of interspinous space estimation. A safe and effective technique should be studied to reduce the misidentification of interspinous level. Two studies suggesting the utility of ultrasound imaging pointed out that they needed to compare ultrasound

Table 2 Comparison of characteristic factors between correct and error cases.

\begin{tabular}{|c|c|c|c|}
\hline & Correct & Error & $\mathrm{p}$ value \\
\hline Total number & $563(67)$ & $272(33)$ & \\
\hline Age & $32(19-48)$ & $32(20-43)$ & $0.57^{*}$ \\
\hline Body Weight (kg) & 60.7 (59-107.2) & $61.1(40.6-105)$ & $0.23^{*}$ \\
\hline Height $(\mathrm{cm})$ & $157(142-173)$ & $157(138-174)$ & $0.28^{\dagger}$ \\
\hline Body Mass Index (kg. m²) & $24.5(15.6-41.9)$ & $24.9(18.3-44.9)$ & $0.15^{*}$ \\
\hline Gestational Age at delivery (week) & $38(25-41)$ & $38(23-42)$ & $0.38^{*}$ \\
\hline \multicolumn{4}{|l|}{ Type of surgery } \\
\hline Planned & $440(67.7)$ & $210(32.3)$ & \multirow{2}{*}{$0.76^{\ddagger}$} \\
\hline Emergency & $123(66.5)$ & $62(33.5)$ & \\
\hline
\end{tabular}

Values are Median (range) or Number (\%); Correct case: Agreement between Palpation and Catheter Insertion.

Error case: Disagreement between Palpation and Catheter Insertion; *: Mann-Whitney-U test; †: t-test; $\ddagger$ : Chi-square test. 
and "gold standard" techniques such as the radiography to validate the use of ultrasound ${ }^{8,10}$. Our study, comparing anesthesiologist's palpation and radiographic images can be an important reference to assess the utility of a new technique in future studies.

Some of the previous studies had evaluated the interspinous level in the postpartum period ${ }^{8,9}$ and another study performed palpation in the sitting position ${ }^{10}$, both of which may not represent the situation of obstetric anesthesia for pregnant woman in labor including cesarean section, especially in Japan. In our study, the anesthesiologist performed every palpation right before the anesthesia and the catheter insertion point does not change after delivery.

This study's limitation is the fact that this was a retrospective study at a single institution. Anesthesiologists' experience varied widely from residents to senior doctors, though all procedures were performed under the supervision of senior doctors.

In conclusion, palpation may not be a reliable technique, with an error of one third to identify the interspinous level in obstetric patients. It seems to be safer for anesthesiologists to aim at L3-4 interspinous level or lower in the intrathecal technique.

\section{References}

1. Reynolds F - Damage to the conus medullaris following spinal anaesthesia. Anaesthesia. 2001;56:238-247.

2. Parry H - Spinal cord damage. Anaesthesia. 2001;56:290.

3. Collier CB, Gatt SP - More reports of spinal cord damage by spinal needles. Anaesth Intens Care. 2002;30:532.

4. levins FA - Accuracy of placement of extradural needles in the L3-4 interspace: comparison of two methods of identifying L4. Br J Anaesth. 1991;66:381-382.
5. Van Gessel EF, Foster A, Gamulin Z - Continuous spinal anesthesia: where do spinal catheter go? Anesth Analg. 1993;76:1004-1007.

6. Broadbent CR, Maxwell WB, Ferrie R, Wilson DJ, Gawne-Cain M, Russell R - Ability of anaesthetists to identify a marked lumbar interspace. Anaestehsia. 2000;55:1122-1126.

7. Furness G, Reilly MP, Kuchi S - An evaluation of ultrasound imaging for identification of lumbar intervertebral level. Anaesthesia. 2002;57:277-280.

8. Whitty R, Moore M, Macarthur A - Identification of the lumbar interspinous spaces: palpation versus ultrasound. Anesth Analg. 2008;106:538-540.

9. Schlotterbeck H, Schaeffer R, Dow WA, Touret Y, Bailey S, Diemunsch $P$ - Ultrasonographic control of the puncture level for lumbar neuraxial block in obstetric anaesthesia. $\mathrm{Br} \mathrm{J}$ Anaesth. 2008;100:230-234.

10. Lee AJ, Ranasinghe JS, Chehade JM, Arheart K, Saltzman BS, Penning DH, Birnbach DJ - Ultrasound assessment of the vertebral level of the intercristal line in pregnancy. Anesth Analg. 2011;113:559-564.

11. Thomson A - Fifth annual report of the committee of collective investigation of Anatomical Society of Great Britain and Ireland for the Year 1893-1894. J Anat Physiol. 1894;29:35-60.

12. Soleiman J, Dmaerel P, Rocher S, Marchal G - Magnetic resonance imaging study of the level of termination of the conus medullaris and the thecal sac: influence of age and gender. Spine. 2005;30:1875-1880.

13. Kim JT, Bahk JH, Sunf $J$ - Influence of age and sex on the position of the conus medullaris and Tuffier's line in adults. Anesthesiology. 2003;99:1359-1363.

14. Faitot V, Ourchane R, Dahmani S et al. - An observational study of factors leading to difficulty in resident anaesthesiologists identifying the epidural space in obstetric patients. Int J Obstet Anesth 2011;20:124-127. 